\title{
Efficient adhesion of bubble on superhydrophobic tapered groove surface
}

\author{
Jianfeng Tang ${ }^{1}$, Cong Liu ${ }^{1}$, Shile Feng ${ }^{1}$ and Yahua Liu ${ }^{1, *}$ \\ ${ }^{1}$ Key Laboratory for Precision \& Nontraditional Machining Technology of Ministry of Education, Dalian University of Technology, Dalian, \\ Liaoning, 116024, China
}

\begin{abstract}
Efficient adhesion of gas bubbles in aqueous environments has great application potentials in various gas-related processes, especially in wastewater treatment. In previous research, much attention has been paid to the design of underwater bubbles transport platforms, however, the bubble adhesion abilities of them are explored insufficiently. Herein, we investigated the bubble adhesion abilities of the superhydrophobic V-shaped pattern (SVP) and a designed superhydrophobic tapered groove (STG). It is revealed that the STG exhibits efficient bubble adhesion abilities compared with the SVP, including bubble retention time $(t)$, adhesion volume and holding efficiency $(\eta)$. In addition, the STG can retain stable adhesion abilities after several adhesion cycles. We believe that these results provide an alternative approach for subaqueous bubble practical applications.
\end{abstract}

\section{Introduction}

Gas bubbles is ubiquitous in nature and of practical importance in many industrial processes ${ }^{[1-6]}$. For example, aquatic insects are capable of utilizing gas bubbles or the air plastron as a physical gill to survive underwater; under the assistance of gas bubbles adhering on hydrophobic solid surface, valuable minerals can be collected from ores. Recent research revealed that superhydrophobic surfaces exhibit superaerophilicity ${ }^{[7-9]}$, which enables the bubble to stick on it via generating adhesive force to offset the buoyancy ${ }^{[10-12]}$. Based on this, various spontaneous bubble transport strategies have been proposed by mimicking nature or artificial designs ${ }^{[13-15]}$. Long et al. ${ }^{[2]}$ fabricated a multi-bioinspired electrode, which was based on a Janus asymmetric foam with dual gradients, i.e., the wettability gradient to promote the one-way gas penetration and the geometry gradient to boost the spontaneous transport of bubbles in the horizontal direction. In bubble transport process, the adhesion abilities of surfaces are crucial for stable and efficient bubble transport, which is mainly influenced by the surface chemical composition and physical morphology ${ }^{[16-18]}$. Gukeh et al. ${ }^{[10]}$ investigated the influence of wettability-confined tracks on surface adhesion, which can be reflected through bubble morphology and retention time. In some gas bubblerelated processes, especially in wastewater treatment, enough adhesion force is capable of prolonging the time of $\mathrm{O}_{3}$ bubbles retaining in aqueous media and contacting with organic contamination, which is supposed to improve the degradation efficiency, thus improve the wastewater treatment efficiency ${ }^{[19,20]}$. Zhang et al. ${ }^{[11]}$ revealed the influence of superhydrophobic pattern shapes (fivepointed star, triangle, circular, and ellipse) on retention time of underwater bubble, which shows that the retention time and the bubble-holding efficiency grow with the shape of the pattern approaching to circular.

However, the bubble adhesion abilities, including adhesion volume, retention time $t$ and bubble-holding efficiency $\eta$, of bubble transporting platforms are explored insufficiently. In this paper, we fabricated superhydrophobic V-shaped pattern (SVP) and a designed superhydrophobic tapered groove (STG) through methods of micro-milling, low-surface-energy modification, chemical etching and laser ablation processes. The adhesion abilities on the two surfaces are studied. We find the bubble can retain longer time in one adhesion cycle on the STG compared with that on SVP, and the bubble adhesion volume and holding efficiency of the STG significantly are increased with the increase of apex angle of tapered grooves. In addition, the STG can retain stable adhesion abilities after several adhesion cycles. We believe that these results provide an alternative approach for subaqueous bubble practical applications.

\section{Materials and methods}

The tapered groove surface was created based on type $6061 \mathrm{Al}$ alloy plate (Shenzhen Hongwang Mold Co., Ltd., China) with a size of $60 \mathrm{~mm} \times 60 \mathrm{~mm} \times 5 \mathrm{~mm}$ by micromilling method after removing the oxide films with abrasive paper. The apex angle $\alpha$ and channel length $L$ are from $2^{\circ}$ to $10^{\circ}$ and $35 \mathrm{~mm}$, respectively. Then, the asfabricated surface was rinsed with acetone and ethanol, followed by ultrasonic cleaning in deionized water to remove the coolant and debris. To remove the defects coming from micro-milling, the chemical etching process was conducted. In the chemical etching process, the as-

* Corresponding author: yahualiu@dlut.edu.cn 
prepared surface was immersed in a freshly aqueous solution of $4 \mathrm{~mol} \cdot \mathrm{L}^{-1}$ hydrochloric acid for $15 \mathrm{~min}$ followed by thorough rinsing with deionized water and drying with a high-purity nitrogen stream. All of the surfaces were modified by spraying low-surface-energy mixtures followed by drying at room temperature for $1 \mathrm{~h}$ to render surfaces superhydrophobic. To obtain wettabilityconfined structure, the laser ablation method was performed to the peripheral section of the tapered grooves. We choose SVP as controlled samples to demonstrate the advantages of the STG. The surface preparation process includes chemical etching, low-surface-energy modification and laser ablation process.

The surface micro-morphology of the STG was characterized by a scanning electron microscope (SEM, SUPRA 55 SAPPHIRE, Germany). An optical contact angle meter system (OCA25, Dataphysics $\mathrm{GmbH}$, Germany) was used to measure the static contact angle of water and bubble underwater for droplets or bubbles of $\sim 3$ $\mu \mathrm{L}$. At least five individual measurements were conducted on each sample. Bubble transport experiments were implemented at ambient temperature on the STG and SVP
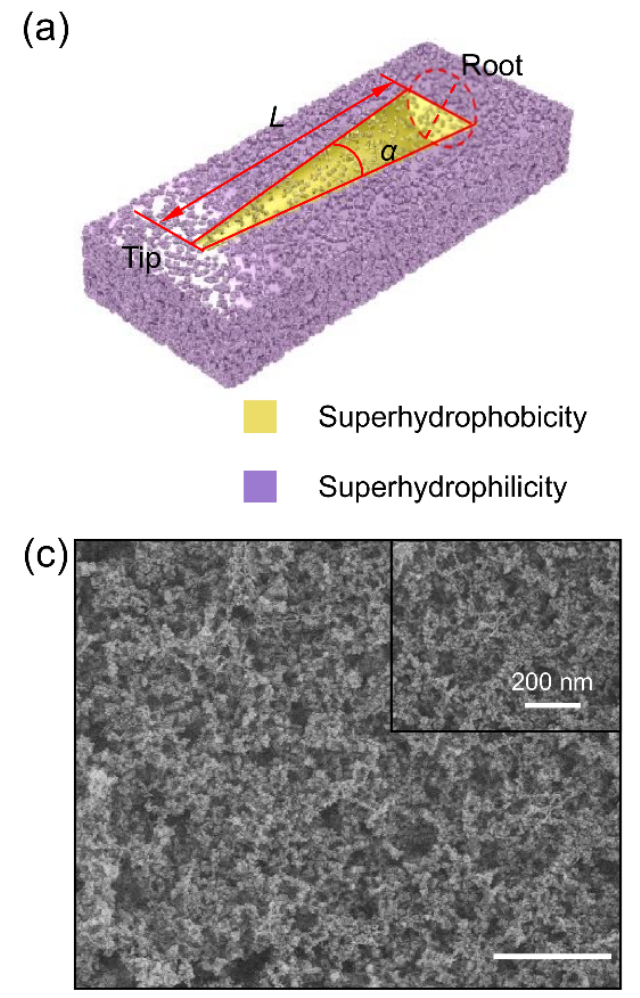

Inside section to measure the adhesion abilities, including bubble retention time $(t)$, adhesion volume and holding-efficiency $(\eta)$. Bubbles were released by a syringe needle at the depth of $20 \mathrm{~mm}$ underwater with an injection velocity of 2 $\mathrm{mL} / \mathrm{min}$ at the tip. The distance between syringe needle tip and the $\mathrm{Al}$ alloy plate was fixed at $1 \mathrm{~mm}$, and the images of accumulated bubbles at the root were recorded by a high-speed camera (250 frames/s, Photron SA4, Japan).

\section{Results and discussion}

\subsection{Characteristics of prepared surfaces}

In the experiments, the fabricated STG is wettabilityconfined structure, which is consisted of a concave superaerophilic groove laid on a superaerophobic substrate (Figure 1a), similar with he SVP. The inside section of the tapered groove is superhydrophobic with water contact angle $\left(\theta_{\mathrm{w}}\right)$ of $\sim 154^{\circ}$ and superaerophilic with bubble contact angle $\left(\theta_{\mathrm{b}}\right)$ of $\sim 0^{\circ}$. The outside section of the tapered groove is superhydrophilic with $\theta_{\mathrm{w}}$ of $\sim 0^{\circ}$ and superareophobic with $\theta \mathrm{b}$ of $\sim 156^{\circ}$ (Figure $1 \mathrm{~b}$ ).
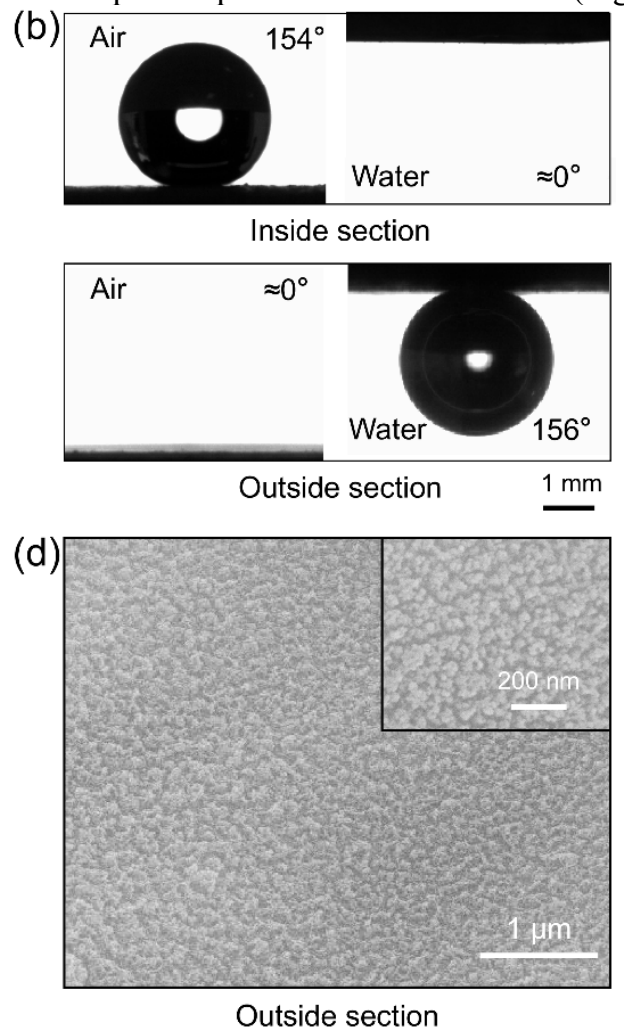

Fig. 1. Characteristics of the STG. (a) Schematic diagram of the STG. (b) The wetting performance of the STG. (c, d) Low- and high-magnified SEM images of the inside and outside section of tapered groove.

To clarify the wetting difference and micromorphology of the surface inside and outside the grove on STG were characterized via scanning electron microscope (SEM), respectively (Figure 1c, d). It was revealed that the surface inside the tapered groove was coated by more nanoparticles compared with the outside. In other words, the sprayed hydrophobic silicon dioxide nanoparticles on surface outside were almost completely removed in laser etching process. Therefore, the apparent wetting difference inside and outside the tapered groove is attributed to the content distinction of hydrophobic silicon dioxide nanoparticles.

\subsection{Bubble retention time}

On the bubble transport platforms, the captured bubbles directionally move from the tip to the root and accumulate there. The accumulated bubbles grow with the continuous transport of bubbles and finally depart from the surface. We define the whole process from the bubble starting 
accumulating at the root and finally departing from the surface as one adhesion cycle. The time bubble retain in one adhesion cycle, which is defined as retention time $(t)$, at the root on the SVP and STG has not revealed so far. To record the $t$ in one adhesion cycle, the SVP and the STG with $\alpha$ and $L$ of $2^{\circ}$ and $35 \mathrm{~mm}$ were placed underwater at room temperature $\left(\sim 25^{\circ} \mathrm{C}\right)$. The bubbles are generated via a syringe needle with injection velocity of $2 \mathrm{~mL} / \mathrm{min}$.

In one adhesion cycle, the accumulated bubbles at the (a) root becomes bigger and exhibits distinction in bubble morphology with the continuous transport of bubbles, as shown in Figure 2. On the STG, the accumulated bubble locating at the root performs a tabular morphology, however, the morphology of bubble at the root on the SVP is more bumped at anytime. Meanwhile, it can be seen that the accumulated bubble on the STG can retain more time compared with SVP, which is crucial in the process of wastewater treatment.

STG

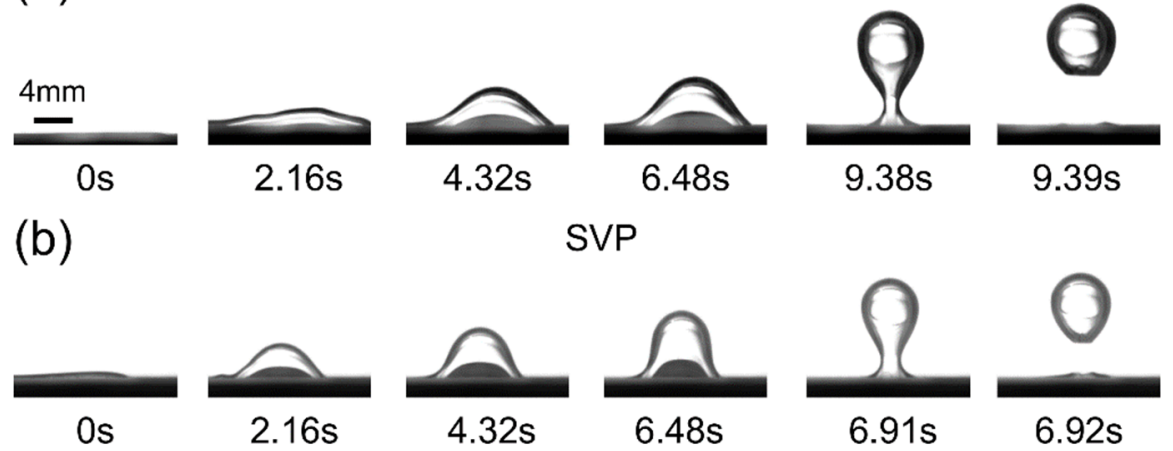

Fig. 2. Image sequences of subaqueous bubble morphology at the root of the STG (a) and the SVP (b) in the whole transport process.

Further, we recorded the bubble retention time $(t)$ on the SVP and STG with different apex angle $\alpha$ (Figure 3a). With the increase of apex angle $\alpha$ from $2^{\circ}$ to $10^{\circ}$, the two surfaces can both retain underwater bubbles for increased time and the retention time $t$ on the STG is always longer

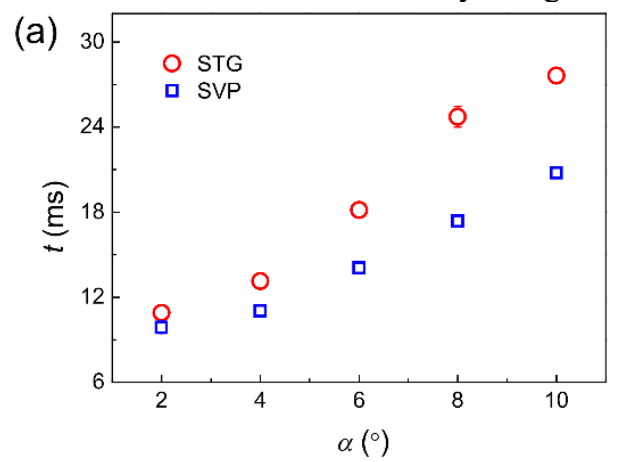

than that on the SVP. To measure the adhesion stability of the STG, we investigate $t$ in a long-time range (Figure $3 b$ ). The result indicates that the STG can retention bubble for stable time even after several adhesion cycles.

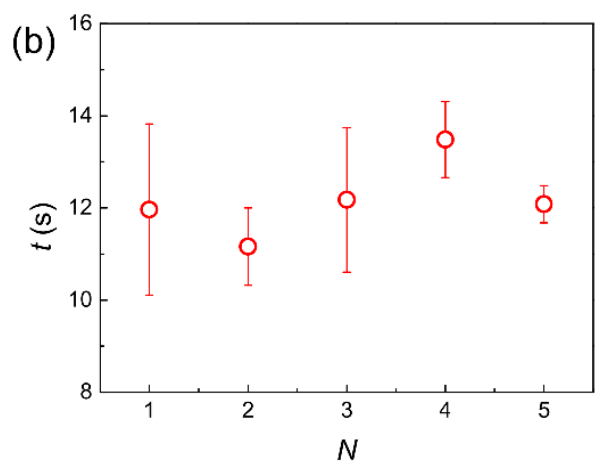

Fig. 3. Influence of apex angle $\alpha$ (a) and adhesion cycle $(N)$ on bubble retention time $(t)$ of the SVP and STG.

\subsection{Bubble adhesion}

Many biological surfaces in nature can effectively adsorb bubbles in the liquid environment through micro-nano hierarchical structures and special chemical components, and it is of great significance to increase the adhesion volumes of bubbles that can be adhered to underwater surfaces. For example, taking advantages of the superhydrophobic properties of abdomen, the water spider (Argyroneta aquatica), and the great diving beetle (Dytiscus marginalis) could efficiently hold gas bubble or air plastron underwater. Therefore, the bubble adhesion volume and bubble efficiency $(\eta)$ were also revealed of the

\section{SVP and STG.}

The adhesion volume is characterized by the bubble diameter $(D)$ when departing from the root. Figure 4 shows the influence of the apex angle $\alpha$ and adhesion cycle $N$ on $D$ on the two surfaces. The optical images of the bubble departing from the root exhibit different $D$, as shown in Figure 4a. It is found that when $\alpha$ is same, $D$ on the STG is larger than that on the SVP, and $D$ increases with the increase of $\alpha$ (Figure 4b). The adhesive force $F_{\mathrm{A}}$ can be deduced by $F_{\mathrm{A}}=\rho \mathrm{g} V$, which is equivalent by the buoyancy, therefore, the STG can enhance the surface adhesive force and bubble adhesion volume. Meanwhile, the results indicate that the adhesion volume maintain stable in various cycles $(N)$ (Figure 4c, d). 
(a)

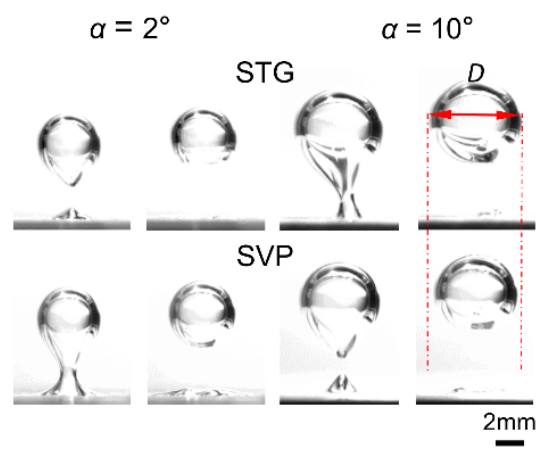

(c)

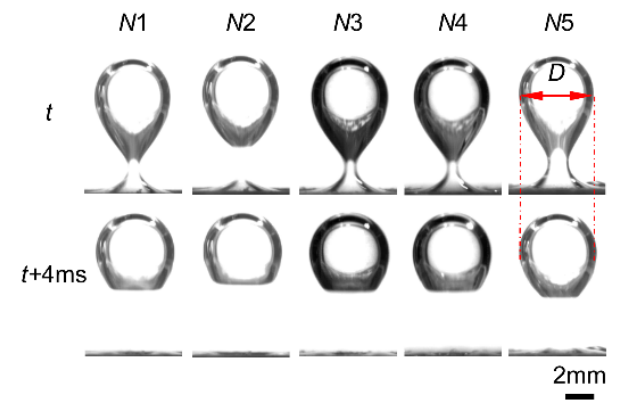

(b)

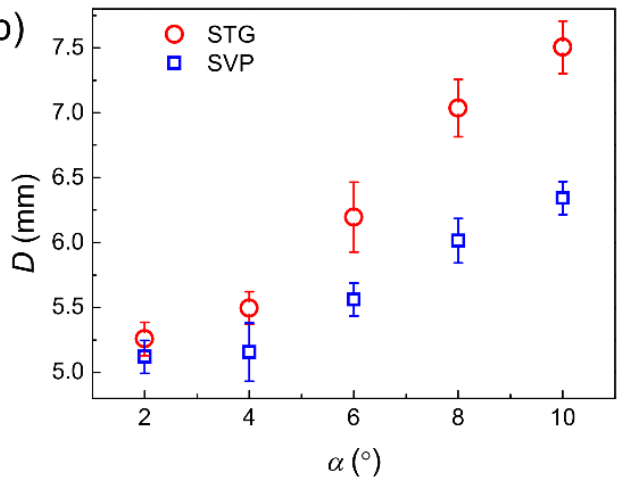

(d)

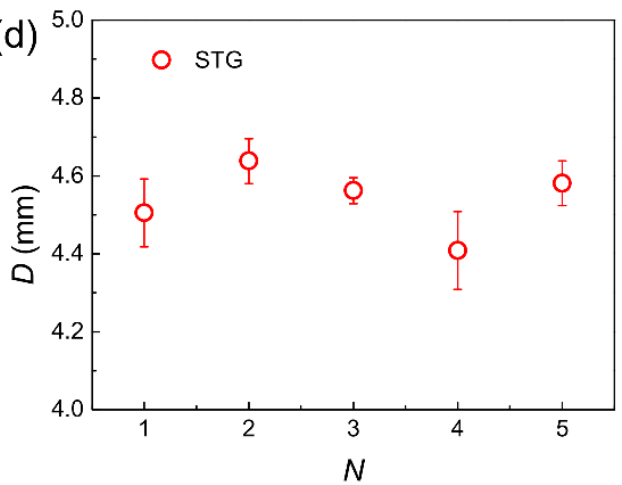

Fig. 4. Influence of the apex angle $\alpha$ and the adhesion cycle $(N)$ on bubble departure diameter $(D)$ of the SVP and STG. (a,b) Optical images and $D$ of bubbles leaving from the SVP and STG with different apex angles. (c,d) Optical images and $D$ of bubbles leaving from the STG after different adhesion cycles.

Further, bubble-holding efficiency $(\eta)$ are also recorded, as shown in Figure 5, which are important in the wastewater treatment process. Taking the contact area into consideration, we simultaneously estimate the bubbleholding efficiency $(\eta)$ on the two surfaces. The bubbleholding efficiency $(\eta)$ can be deduced by $\eta=\mathrm{m} / \mathrm{A}$, in which $\mathrm{m}$ is the mass of held gas bubble ( $\mathrm{mg}$ ) and $\mathrm{A}$ is the contact area between bubble and surface $\left(\mathrm{cm}^{2}\right)$. It is found that on the SVP, $\eta$ is $\sim 0.16 \mathrm{mg} / \mathrm{cm}^{2}$ with $\alpha$ of $2^{\circ}$, and decreases to a lower value of $\sim 0.12 \mathrm{mg} / \mathrm{cm}^{2}$ with $\alpha$

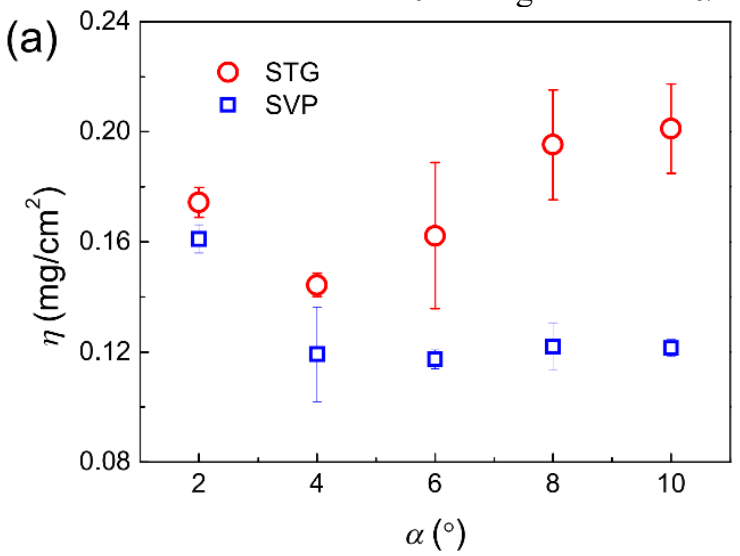

ranging from $4^{\circ}$ to $10^{\circ}$. However, on the STG, the $\eta$ decreases a little with $\alpha$ changing from $2^{\circ}$ to $4^{\circ}$, and then increases gradually with the increase of $\alpha$ (Figure 5a). The Figure $5 \mathrm{~b}$ shows that the bubble-holding efficiency $(\eta)$ on STG with $\alpha$ of $2^{\circ}$ maintains stable in various cycles $(N)$. These results indicate that the STG has higher $\eta$ compared with the SVP and manifests robust stability of adhesion abilities, which is more potential in bubble-related processes.

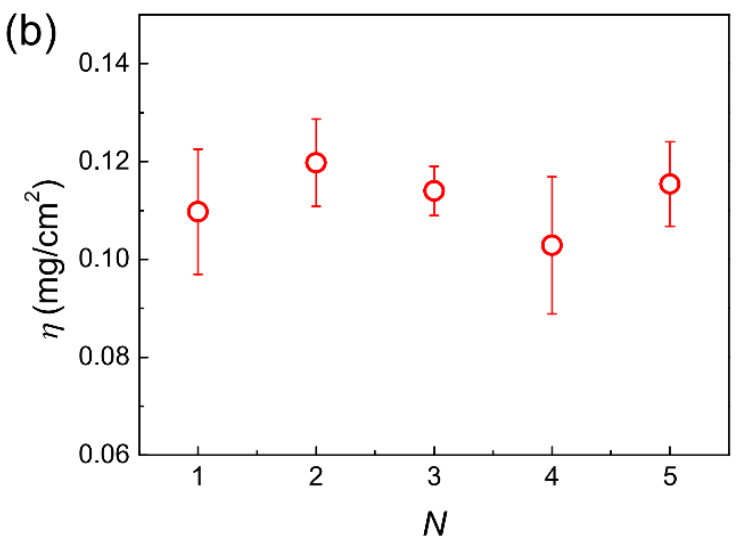

Fig. 5. Influence of the apex angle $\alpha$ (a) and the adhesion cycle $N(\mathrm{~b})$ on the bubble-holding efficiency $(\eta)$ of the STG and SVP.

\section{Conclusion}

Overall, we have utilized methods of micro-milling, lowsurface-energy modification and laser ablation processes to fabricate superhydrophobic V-shaped pattern (SVP) and a designed superhydrophobic tapered groove (STG) on the superhydrophilic substrate. Compared with the SVP, the STG manifests excellent bubble adhesion abilities, including bubble retention time $t$, adhesion volumes, and holding-efficiency $\eta$ under the same structural parameter. In addition, the STG can maintain robust stability of adhesion abilities. Based on these results, we envision that this multifunctional bubble manipulation system will be 
applied to various practical applications, such as wastewater treatment, heat transfer and chemical engineering.

\section{Acknowledgments}

We are grateful for the support from National Natural Science Foundation of China (52075071, 51605073, 52005075), the Fundamental Research Funds for the Central Universities (DUT18RC(3)048, DUT19RC(3)055), Liao Ning Revitalization Talents Program (XLYC1807092) and Opening Project of the Key Laboratory of Bionic Engineering (Ministry of Education), Jilin University (KF20200002). Y. L. and S. F. acknowledge support from the Star Ocean Outstanding Talents Program.

\section{References}

1. G. O. Stride, Nature, 171, 885 (1953)

2. Z. Long; Y. Zhao; C. Zhang; Y. Zhang; C. Yu; Y. Wu; J. Ma; M. Cao; L. Jiang, Adv. Mater., 32, 1908099 (2020)

3. C. Yu; M. Cao; Z. Dong; K. Li; C. Yu; J. Wang; L. Jiang, Adv. Funct. Mater., 26, 6830 (2016)

4. C. Pei; Y. Peng; Y. Zhang; D. Tian; K. Liu; L. Jiang, ACS Nano, 12, 5489 (2018)

5. R. Ma; J. Wang; Z. Yang; M. Liu; J. Zhang; L. Jiang, Adv. Mater., 27, 2384 (2015)

6. M. Sarkar; S. Donne; G. Evans, Adv. Powder Technol., 21, 412 (2010)

7. J. Wang; Y. Zheng; F. Nie; J. Zhai; L. Jiang, Langmuir, 25, 14129 (2009)

8. H. Yang; J. Hou; L. Wan; V. Chen; Z. Xu, Advanced Materials Interfaces, 3, 1500774 (2016)

9. J. Yong; F. Chen; Y. Fang; J. Huo; Q. Yang; J. Zhang; H. Bian; X. Hou, ACS Appl. Mater. Inter., 9, 39863 (2017)

10. M. Jafari Gukeh; T. Roy; U. Sen; R. Ganguly; C. M. Megaridis, Langmuir, 36, 11829 (2020)

11. C. Zhang; M. Cao; H. Ma; C. Yu; K. Li; C. Yu; L. Jiang, Adv. Funct. Mater., 27, 1702020 (2017)

12. T. A. Oratis; M. W. J. Bush; A. H. Stone; C.J. Bird, Science (American Association for the Advancement of Science), 369, 685 (2020)

13. Z. Zheng; H. Yang; Y. Cao; Z. Dai, ACS Omega, 5, 718 (2020)

14. K. Sun; H. Yang; W. Xue; M. Cao; K. Adeyemi; Y. Cao, Langmuir, 34, 13203 (2018)

15. B. Chen; T. Wada; H. Yabu, Langmuir, 36, 6365 (2020)

16. F. Geyer; C. Schönecker; H. Butt; D. Vollmer, Adv. Mater., 29, 1603524 (2017)

17. Y. Jiao; C. Li; X. Lv; Y. Zhang; S. Wu; C. Chen; Y. $\mathrm{Hu}$; J. Li; D. Wu; J. Chu, J. Mater. Chem. A, 6, 20878 (2018)

18. D. Gao; J. Cao; Z. Guo, Chemical communications
(Cambridge, England), 55, 3394 (2019)

19. W. Barthlott; T. Schimmel; S. Wiersch; K. Koch; M. Brede; M. Barczewski; S. Walheim; A. Weis; A. Kaltenmaier; A. Leder, Adv. Mater., 22, 2325 (2010)

20. H. S. Huynh; Y. C. Lau; H. B. Cheong; M. Muradoglu; W. O. Liew; W. T. Ng, Soft Matter, 11, 7474 (2015) 